\title{
AUTISMO E SÍNDROME DE DOWN: CONCEPÇÕES DE PROFISSIONAIS DE DIFERENTES ÁREAS ${ }^{1}$
}

\author{
Cibele Shírley Agripino-Ramos² \\ Nádia Maria Ribeiro Salomão \\ Universidade Federal da Paraíba, João Pessoa-PB, Brasil
}

\begin{abstract}
RESUMO. Este estudo teve como objetivo analisar as concepções de profissionais de diferentes áreas acerca do autismo e da síndrome de Down, tanto daqueles com experiência quanto dos sem experiência no trabalho com esses indivíduos. Participaram 75 profissionais, distribuídos nos seguintes grupos: psiquiatras, neurologistas, pediatras, fonoaudiólogos, fisioterapeutas, terapeutas ocupacionais, educadores físicos, psicólogos, pedagogos e professores. Foram realizadas entrevistas semiestruturadas com os participantes, as quais foram transcritas literalmente e submetidas à técnica de Análise de Conteúdo, proposta por Bardin. Ao analisar os relatos dos profissionais, percebeuse que aqueles com experiência apresentaram conhecimentos mais específicos sobre o autismo e a síndrome de Down, enquanto aqueles sem experiência frequentemente mencionavam características mais conhecidas das duas síndromes. Embora tenham considerado os prejuízos que podem estar presentes no autismo e na síndrome de Down, observou-se que, de uma forma geral, os profissionais demonstraram também ter concepções positivas em relação a essas duas condições, ao reconhecerem as capacidades que tais indivíduos podem desenvolver. Destacou-se o fato de alguns profissionais desconhecerem o autismo, o que não ocorreu em relação à síndrome de Down, o que indica a necessidade de uma maior capacitação dos profissionais, sobretudo quanto à primeira dessas condições, vindo a contribuir para a inclusão escolar e social desses indivíduos.
\end{abstract}

Palavras-chave: Autismo; síndrome de Down; concepções.

\section{AUTISM AND DOWN SYNDROME: CONCEPTIONS OF PROFESSIONALS OF DIFFERENT AREAS}

\begin{abstract}
This study aimed to analyze the conceptions of professionals from different areas about autism and Down syndrome, those with and without experience in working with these individuals. It included 75 professionals distributed in the following groups: psychiatrists, neurologists, pediatricians, speech therapists, physiotherapists, occupational therapists, physical educators, psychologists, pedagogues and teachers. Semi-structured interviews were conducted with the professionals, which were literally transcribed and submitted to the technique Content Analysis, proposed by Bardin. By analyzing the reports of professionals, it was found that those with experience had more specific knowledge about autism and Down syndrome, while those without experience often mentioned more general features of the two syndromes. Although they considered the deficits that may be present in autism and Down syndrome, it was observed that, in general, the professionals also have demonstrated positive conceptions regarding these two conditions, recognizing the capacities of these individuals. It was noted that some professionals had no knowledge about autism, which did not occur in relation to Down syndrome, pointing to the need for better training of professionals, especially in relation to the first of these conditions, in order to contribute to school and social inclusion of these individuals.
\end{abstract}

Keywords: Autism; Down syndrome; conceptions.

\section{AUTISMO Y SÍNDROME DE DOWN: CONCEPCIONES DE PROFESIONALES DE DIFERENTES ÁREAS}

RESUMEN. Este estudio tuvo como objetivo analizar las concepciones de profesionales de diferentes áreas sobre el autismo y el síndrome de Down, tanto de aquellos con experiencia como los sin experiencia en el trabajo con

\footnotetext{
1 Apoio e financiamento: Coordenação de Aperfeiçoamento de Pessoal de Nível Superior (CAPES).

2 Endereço para correspondência: Rua José Gomes da Silveira, 500, Cristo Redentor,CEP 58070-390, João PessoaPB. E-mail: cibeleagripino@yahoo.com.br
} 
estos individuos. Participaron 75 profesionales distribuidos en los siguientes grupos: psiquiatras, neurólogos, pediatras, fonoaudiólogos, fisioterapeutas, terapeutas ocupacionales, educadores físicos, psicólogos, pedagogos y profesores. Se realizaron entrevistas semiestructuradas con los participantes, las cuales fueron transcriptas literalmente y sometidas a la técnica de Análisis de Contenido, propuesta por Bardin. Al analizar los relatos de los profesionales, se percibió que aquellos con experiencia presentaron conocimientos más específicos sobre el autismo y el síndrome de Down, mientras que los que no tenían experiencia frecuentemente mencionaban características más conocidas de los dos síndromes. Aunque hayan considerado los perjuicios que pueden estar presentes en el autismo y en el síndrome de Down, se observó que, en general, los profesionales demostraron también tener concepciones positivas con respecto a estas dos condiciones, al reconocer las capacidades que estos individuos pueden desarrollar. Se destacó el hecho de que algunos profesionales desconocen el autismo, lo que no ocurrió con relación al síndrome de Down, lo que apunta para la necesidad de una mayor capacitación de los profesionales, sobre todo en cuanto a la primera de estas condiciones, contribuyendo, así, para la inclusión escolar y social de estos individuos.

Palabras clave: Autismo; síndrome de Down; concepciones.

O autismo é um transtorno do desenvolvimento que vem sendo bastante estudado em diversos campos do saber. Observa-se que muitos estudos a seu respeito procuram comparar algum aspecto relacionado a esse transtorno com a síndrome de Down ou com o desenvolvimento típico. Os estudos comparativos do autismo e da síndrome de Down, especificamente, têm sido realizados com o intuito de conhecer a dinâmica familiar presente nos dois grupos (Griffith, Hastings, Nash, \& Hill, 2010; Pisula, 2007), bem como o sistema de crenças dos genitores desses indivíduos (King, Baxter, Rosenbaum, Zwaigenbaum, \& Bates, 2009; King et al., 2006).

Com relação aos estudos que procuram investigar as concepções de profissionais sobre essas duas síndromes, verifica-se que eles têm se voltado mais para os profissionais da área da Educação, mas mesmo ainda assim, são poucos esses estudos (Goldberg, 2002; York, Fraunhofer, Turk, \& Sedgwick, 1999). Nesse sentido, considerando a importância tanto dos profissionais da área da Educação quanto dos da Saúde no atendimento a pessoas diagnosticadas dentro do espectro autista e àquelas com síndrome de Down, o presente estudo buscou investigar as concepções de profissionais de diferentes áreas do conhecimento acerca dessas duas condições, tanto daqueles com experiência quanto dos sem experiência no trabalho com tais indivíduos.

Destaca-se que a incidência do autismo e da síndrome de Down tem sido considerada relativamente alta nos dias de hoje (BaronCohen, 2008; Melo-de-Aguiar, 2009), o que reforça a necessidade de se conhecer o que os profissionais sabem a respeito dessas duas condições. Além disso, estudar as concepções de profissionais acerca do autismo e da síndrome de Down implica também levar em consideração algumas particularidades relacionadas a cada uma dessas síndromes.

A primeira delas diz respeito ao período em que é feita a identificação, visto que a síndrome de Down pode ser identificada logo após o nascimento da criança (Henn, Piccinini, \& Garcias, 2008), enquanto o autismo costuma ser diagnosticado somente por volta dos três anos de vida, embora seja enfatizada atualmente a necessidade de uma identificação precoce desse transtorno (Lampreia \& Lima, 2008). Outro aspecto que se destaca refere-se ao funcionamento cognitivo presente nos dois grupos, pois geralmente os indivíduos com síndrome de Down apresentam deficiência mental variável (Dumas, 2011), ao passo que o autismo pode estar associado ou não à deficiência mental (Klin, 2006).

Além disso, partindo-se de uma perspectiva que considera a importância das interações sociais para o desenvolvimento infantil, pode ainda se referir a sociabilidade no caso do autismo e no da síndrome de Down: enquanto o autismo tem como uma de suas características centrais um prejuízo social - embora se reconheça que, por se tratar de um espectro de condições, pode haver variabilidade quanto à sociabilidade (Mitchell \& O'Keefe, 2008) -, os indivíduos com síndrome de Down frequentemente são descritos como sociáveis e afetuosos, apesar de essa noção ser contestada por alguns autores (Rodrigues, Alchieri, \& Coutinho, 2010). De todo modo, considerou-se que tais aspectos poderiam levar os profissionais a diferentes concepções sobre essas pessoas.

Ressalta-se também, no presente estudo, a influência das concepções nas interações sociais 
e no comportamento. Em relação a esse aspecto, partiu-se da ideia de que a forma como os profissionais concebem essas síndromes influencia nas estratégias de intervenção que serão adotadas e determina o tipo de informação que serão transmitidos aos pais de tais indivíduos, além de trazer implicações importantes para as discussões acerca da inclusão de crianças e jovens com necessidades educativas especiais.

\section{MÉTODO}

Esta pesquisa se caracteriza por uma abordagem metodológica qualitativa e quantitativa.

\section{Participantes}

Participaram deste estudo 75 profissionais, a saber, cinco psiquiatras, seis neurologistas e oito profissionais de cada um dos seguintes grupos: pediatras, fonoaudiólogos, fisioterapeutas, terapeutas ocupacionais, educadores físicos, psicólogos, pedagogos e professores. Os participantes foram, em sua maioria, do sexo feminino - $76 \%$ deles - e a idade variou entre 23 e 62 anos.

Os profissionais trabalhavam em instituições públicas e privadas que atendem pessoas com necessidades educativas especiais em clínicaescola, hospital universitário, hospital psiquiátrico, hospital infantil, Centro de Atenção Psicossocial Infantil (CAPSi), consultórios particulares e ainda em escolas públicas e privadas da rede regular de ensino, todos localizados na cidade de João Pessoa-PB. Apenas um dos profissionais - uma pedagoga atuava em ambiente domiciliar na cidade de Campina Grande-PB.

Do total de participantes, 54,7\% tinham experiência no atendimento a indivíduos tanto com autismo quanto com síndrome de Down; $21,3 \%$ já haviam trabalhado apenas com pessoas com síndrome de Down; e 13,3\% tinham experiência apenas com autismo. Por sua vez, 10,7\% deles não tinham experiência no trabalho com pessoas nem com autismo nem com síndrome de Down.

De todos os profissionais com quem se entrou em contato, sete não aceitaram participar. Foram eles: duas neurologistas, uma pediatra, uma psiquiatra, uma pedagoga, um psicólogo e uma fonoaudióloga. A justificativa mais recorrente foi a indisponibilidade de tempo. Destacou-se a justificativa dada pelo psicólogo e pela fonoaudióloga, os quais afirmaram trabalhar apenas com indivíduos com síndrome de Down, e por isso não responderiam a questões referentes ao autismo.

\section{Instrumentos}

Foi utilizado um questionário sociodemográfico contendo dados como sexo, idade, escolaridade, formação profissional, tempo de formação e local de trabalho, sendo realizada também uma entrevista semiestruturada. A entrevista abordou questões referentes à caracterização do autismo e da síndrome de Down, ao desenvolvimento desses indivíduos, às formas de intervenção a serem utilizadas, à inserção dos indivíduos com essas síndromes em escolas regulares e aos principais desafios relacionados ao trabalho com esses indivíduos e com suas famílias. Para registrar os dados do questionário e da entrevista, foi utilizado um minigravador.

\section{Procedimentos para coleta e análise dos dados}

Após aprovação pelo Comitê de Ética em Pesquisa do Centro de Ciências da Saúde (CEP/CCS) da Universidade Federal da Paraíba, sob o protocolo $n^{\circ}$. 0219/11, o estudo teve início a partir do contato com os responsáveis pelas instituições já mencionadas e com os profissionais que atuavam em consultórios particulares. Nesse primeiro momento foi entregue a cada um uma carta de apresentação, visando a obter uma autorização para realizar a pesquisa, agendar a aplicação do questionário e a entrevista com os profissionais. Foi-lhes solicitado que assinassem um termo de consentimento para a realização do estudo e de permissão para a gravação da entrevista.

As entrevistas foram realizadas, em sua maioria, no ambiente de trabalho de cada profissional, tendo duração média de 20 minutos. Durante as entrevistas, estiveram presentes apenas o participante e a pesquisadora. Posteriormente, as entrevistas foram transcritas de maneira literal e submetidas à técnica de Análise de Conteúdo Categorial-Temática, proposta por Bardin (1977).

Com a análise, foram estabelecidas cinco categorias, quais sejam: caracterização, desenvolvimento, intervenção, inclusão escolar e desafios. A análise também permitiu a composição de subcategorias, sendo importante 
mencionar que elas não são excludentes, o que implica dizer que as respostas dadas por um mesmo profissional podem estar situadas em mais de uma subcategoria.

\section{RESULTADOS E DISCUSSÃO}

\section{Caracterização do autismo e da síndrome de Down}

No que diz respeito à caracterização do autismo e da síndrome de Down, parte dos profissionais se referiu à etiologia das duas condições, sobretudo aqueles que tinham experiência no trabalho com tais indivíduos. Sendo assim, $65 \%$ do total de profissionais entrevistados afirmaram que a síndrome de Down seria causada por fatores genéticos, o que foi relatado principalmente pelos psicólogos e fisioterapeutas. Quanto ao autismo, apenas 12\% dos participantes referiram-se à etiologia do transtorno. Esses participantes, que eram das áreas de Fonoaudiologia, Fisioterapia, Terapia Ocupacional e da área médica, apontaram o envolvimento de fatores neurológicos e genéticos, como também de etiologias múltiplas. Ressalta-se que tais relatos estão de acordo com a literatura, visto que a síndrome de Down envolve, comprovadamente, fatores genéticos e que, atualmente, muitos tendem a conceituar o autismo como uma síndrome de etiologias múltiplas, tendo os estudos apontado peculiaridades nas áreas do funcionamento cerebral e da genética, embora ainda não haja um marcador biológico definitivo (Camargo \& Bosa, 2009; Melo-de-Aguiar, 2009).

O déficit na comunicação/linguagem no autismo foi referido por $25 \%$ dos profissionais, dos quais a maioria era formada por pediatras, fonoaudiólogos e professores, com ou sem experiência. No caso da síndrome de Down, essa característica foi citada por $37 \%$ dos participantes, principalmente dentre aqueles que tinham experiência e que eram das áreas da Fonoaudiologia e Pedagogia.

Também foi mencionada, principalmente por profissionais experientes no trabalho com pessoas com autismo e com síndrome de Down, a possibilidade de que elas apresentem um déficit motor, sobretudo aquelas com síndrome de Down. No caso desta síndrome, tal característica foi referida por $52 \%$ dos participantes, mais frequentemente por fisioterapeutas e fonoaudiólogos, enquanto apenas 9,3\% dos participantes - em sua maioria, fisioterapeutas - citaram esse déficit no caso do autismo.

Ainda quanto à caracterização do autismo e da síndrome de Down, parte dos profissionais mencionou a presença de agressividade. Quanto ao autismo, esse aspecto foi referido por $29 \%$ dos profissionais, tanto com experiência quanto sem experiência, sendo a maior parte deles fisioterapeutas, pediatras, pedagogos e fonoaudiólogos. Destaca-se que os profissionais que já tinham trabalhado com autistas, em geral, citavam a agressividade como algo que não necessariamente caracteriza o autismo, mas que pode ocorrer também em meio a determinadas circunstâncias ambientais. Os profissionais que nunca haviam trabalhado com pessoas com autismo geralmente citavam o comportamento agressivo como característico dessa condição.

A presença de agressividade em indivíduos com síndrome de Down foi citada por $21 \%$ dos participantes, em sua maioria, aqueles que já trabalharam com tais indivíduos e que eram pedagogos. Considera-se importante esclarecer que parte desses profissionais referiu-se à presença da agressividade isoladamente, enquanto outros mencionaram a alternância de comportamentos afetuosos e agressivos ou a variação de humor.

Os profissionais referiram-se também ao funcionamento cognitivo dos indivíduos pertencentes a esses dois grupos. Desse modo, a presença de um déficit cognitivo na síndrome de Down foi mencionada pela maioria dos profissionais dentro de todas as áreas, afora os professores, que mencionaram esse aspecto com menor frequência. Essa subcategoria, que foi mais frequente dentre os profissionais com experiência no atendimento a pessoas com síndrome de Down, foi referida por $68 \%$ dos participantes do estudo.

Salienta-se ainda que, apesar de a literatura afirmar que há uma deficiência mental variável na síndrome de Down, geralmente em nível moderado (Dumas, 2011; Nunes \& Dupas, 2011; Reilly, 2009), um educador físico, um fisioterapeuta e uma psicóloga afirmaram que os indivíduos com síndrome de Down possuem boa capacidade intelectual. Esses profissionais relataram que tais pessoas são capazes de compreender aquilo que Ihes é solicitado e de se expressar adequadamente.

Quanto ao autismo, a presença de um déficit cognitivo foi mencionada por $21 \%$ dos 
participantes, a maioria formada por neurologistas, fonoaudiólogos e psiquiatras, e por aqueles com experiência no trabalho com autistas. Em contrapartida, $8 \%$ do total de profissionais entrevistados - metade deles com experiência com autismo e a outra metade sem essa experiência - afirmaram que os autistas têm uma grande capacidade intelectual. Tais profissionais, que eram das áreas da Fonoaudiologia, Pediatria e Fisioterapia, referiram-se a habilidades em determinadas áreas, como raciocínio lógico e memória; contudo, estima-se que em torno de 60 a $70 \%$ das pessoas com autismo apresentam deficiência mental e que apenas cerca de $10 \%$ possuem um elevado desempenho em alguma habilidade específica (Klin, 2006). Pode ser que esses profissionais tenham dificuldade em diferenciar o autismo da síndrome de Asperger, pois, embora esta síndrome faça parte do espectro autista, um dos pontos em que ela se diferencia do autismo clássico é o de que os indivíduos com síndrome de Asperger, de modo geral, apresentam uma inteligência acima da média (Baron-Cohen, 2008).

Apesar de terem mencionado as características citadas acima, os profissionais afirmaram perceber uma variabilidade nos quadros clínicos, no que se refere tanto ao autismo quanto à síndrome de Down. Tal subcategoria, que foi identificada principalmente nos relatos dos profissionais com experiência no trabalho com esses grupos de indivíduos, foi referida, no que diz respeito ao autismo, por $48 \%$ dos participantes, estando mais presente nos relatos dos psiquiatras, dos terapeutas ocupacionais e dos fisioterapeutas; já no caso da síndrome de Down, foi citada por $49 \%$ dos profissionais, sendo mais frequente entre os pedagogos.

Ainda foi mencionada por parte dos profissionais uma associação com outras condições. Quanto ao autismo, foi referida uma associação com o Transtorno de Déficit de Atenção e Hiperatividade (TDAH) e com a psicose, aspecto mencionado por $11 \%$ dos participantes, com ou sem experiência. Dentre os profissionais que caracterizaram o autismo por meio de uma associação com a psicose, um neurologista e uma psiquiatra, através de seus relatos, pareceram conceber que haveria um continuum entre o autismo e a esquizofrenia. Nesse ponto, os resultados deste estudo assemelham-se aos das investigações realizadas por Heidgerken, Geffken, Modi e Frakey (2005) e Imran et al. (2011), os quais verificaram certa tendência dos profissionais da área médica a concordar com a ideia de que crianças com autismo se tornariam esquizofrênicas na idade adulta. Não obstante, enquanto o autismo se manifesta nos primeiros três anos de vida e tem um curso clínico persistente, o surgimento da esquizofrenia se dá em uma idade mais tardia, dificilmente antes dos sete anos, e, além disso, seu curso não é persistente, e sim episódico, isto é, com melhoras e recaídas (Lampreia \& Lima, 2008).

Foi também referida por dois educadores físicos uma possível associação da síndrome de Down com o autismo, sendo que esses profissionais tinham experiência no atendimento tanto a autistas quanto a pessoas com síndrome de Down. De acordo com a literatura (Castro, Panhoca, \& Zanolli, 2011; Reilly, 2009), há um subgrupo de pessoas com síndrome de Down que pode fazer parte do espectro autista, embora seja difícil determinar a exata prevalência da associação entre as duas síndromes.

No que se refere especificamente ao autismo, $20 \%$ dos profissionais definiram-no como um transtorno do desenvolvimento, sendo a maioria deles psicólogos e psiquiatras experientes no atendimento a pessoas com autismo. Tal definição é condizente com a literatura, visto que, a partir da década de 1980, o autismo passou a ser considerado um Transtorno Global do Desenvolvimento ou Transtorno Invasivo do Desenvolvimento, pelo fato de que ele faz parte de uma família de distúrbios da socialização com início precoce e atinge múltiplas áreas do desenvolvimento (Klin \& Mercadante, 2006).

O comprometimento em importantes áreas do desenvolvimento presente no autismo é comumente denominado de tríade de prejuízos, que se refere aos prejuízos na comunicação verbal e não verbal, na interação social e de falta de flexibilidade mental e comportamental (Lampreia \& Lima, 2008), aspecto que foi mencionado por $45 \%$ dos participantes, os quais, em sua maioria, tinham experiência com autismo. Esses profissionais eram, em sua maioria, psicólogos, terapeutas ocupacionais, psiquiatras e fonoaudiólogos.

Outros profissionais abordaram essas três características separadamente, descrevendo o autismo por meio de uma ou duas delas. Sendo assim, além do déficit na 
comunicação/linguagem

anteriormente, $49 \%$ dos participantes caracterizaram o autismo pela apresentação de dificuldades relacionadas à interação, o que foi relatado dentro de todas as áreas, mas principalmente pelos fisioterapeutas, pelos professores e pelos educadores físicos, e foi relativamente frequente entre aqueles sem experiência.

Também houve relatos sobre a presença de problemas comportamentais no autismo, como os movimentos estereotipados e a resistência a mudanças. Esses aspectos foram mencionados por dois pediatras, uma pedagoga, uma professora e um fisioterapeuta, quatro dos quais tinham experiência e um não tinha experiência com autismo.

Considera-se importante referir ainda o desconhecimento sobre o autismo por parte de três profissionais. Esses participantes, que eram duas professoras e um educador físico, afirmaram não ser capazes de identificar nenhuma característica do transtorno.

No que diz respeito especificamente à síndrome de Down, a presença de características físicas foi destacada por $73 \%$ dos profissionais. Esse foi um aspecto referido dentro de todas as especialidades, por profissionais tanto com quanto sem experiência no atendimento a esses indivíduos, sendo mais frequente entre os professores, os pediatras e os neurologistas. Também foi mencionada a presença de problemas físicos como tendência à obesidade, problemas cardíacos, respiratórios, gastrointestinais, articulatórios e outros, por $44 \%$ dos participantes, os quais, em sua maior parte, tinham experiência com síndrome de Down e eram, sobretudo, profissionais da área médica.

Embora alguns participantes tenham citado uma alternância de comportamentos afetuosos e agressivos em indivíduos com síndrome de Down, houve profissionais que caracterizaram a síndrome de Down pela presença isolada de afetuosidade, que foi referida por $35 \%$ dos participantes, em maior frequência por profissionais com experiência no trabalho com essas pessoas, cujas especialidades eram principalmente a Pediatria e a Educação Física. Sobre essa questão se pode referir que, apesar de haver o estereótipo de que pessoas com síndrome de Down têm um bom temperamento, são afetuosas e calmas, alguns autores contestam essa noção (Rodrigues et al., 2010), ao afirmarem que tal concepção não corresponde à realidade, pelo fato de que são pessoas únicas, que apresentam distinções no que se refere à personalidade e ao temperamento, como qualquer outro indivíduo. Ainda quanto à caracterização da síndrome de Down, foi citado o fato de que esta síndrome é mais conhecida e tem tido uma maior visibilidade na mídia, quando comparada ao autismo. Isso foi referido por $15 \%$ dos participantes, mais frequentemente por aqueles com experiência no trabalho com pessoas com síndrome de Down, sendo relatado em maior número pelos psicólogos.

Outro aspecto abordado por uma professora, uma pediatra e uma pedagoga foi a sexualidade das pessoas com síndrome de Down, percebida como sendo "aguçada". A esse respeito, Moreira e Gusmão (2002) ressaltam que comportamentos que denotam uma impulsividade sexual são decorrentes muito mais da história de vida desses indivíduos - marcada por falta de orientações e de estímulos sociais do que pelo fato de terem a síndrome de Down.

\section{Desenvolvimento no autismo e na síndrome de Down}

Ao abordarem o desenvolvimento das pessoas com autismo e com síndrome de Down, considerando-o um desenvolvimento atípico, $27 \%$ dos participantes no caso do autismo e $52 \%$ no tocante à síndrome de Down conceberam o desenvolvimento de tais indivíduos como lento, comprometido ou apenas diferenciado. Tais profissionais eram, sobretudo, das áreas da Fisioterapia e da Neurologia e tinham experiência no atendimento a esses indivíduos.

Apesar de considerarem os prejuízos inerentes ao autismo e à síndrome de Down, os profissionais afirmaram que esses indivíduos podem se desenvolver apesar das suas limitações, o que foi mencionado tanto por aqueles com experiência quanto pelos sem experiência no trabalho com essas pessoas. Esse aspecto foi referido em relação ao autismo por $36 \%$ dos participantes, sobretudo pelos profissionais da Psicologia e da Pedagogia, enquanto para a síndrome de Down tal aspecto foi indicado por $47 \%$ dos participantes, sendo referido com uma frequência maior pelos terapeutas ocupacionais e fisioterapeutas. Percebe-se, assim, que esses participantes apresentaram uma visão positiva em relação a esses indivíduos, isto é, uma visão focada não apenas nos déficits, mas também no 
reconhecimento das capacidades que eles podem apresentar.

A maioria dos profissionais - em torno de $60 \%$ deles - considerou que o desenvolvimento das pessoas com autismo e com síndrome de Down depende de acompanhamento, que deve ser realizado tanto pela família quanto pela escola e os profissionais. Esse aspecto foi mencionado dentro de todas as especialidades, por profissionais tanto com experiência quanto sem experiência no trabalho com tais indivíduos.

Alguns profissionais, sobretudo aqueles com experiência, também consideraram que o desenvolvimento desses indivíduos depende do grau de comprometimento, principalmente do comprometimento cognitivo. Quanto ao autismo, esse aspecto foi mencionado por $19 \%$ dos participantes, que em sua maioria eram psiquiatras, terapeutas ocupacionais e fonoaudiólogos, sendo referido quanto à síndrome de Down por $28 \%$ dos participantes, em sua maioria da área da Fonoaudiologia, os quais apontaram também o comprometimento a nível físico.

No que diz respeito especificamente ao autismo, $9,3 \%$ dos participantes mencionaram que autistas possuem um desenvolvimento físico típico, pelo fato de que, diferentemente da síndrome de Down, no autismo não há uma caracterização física. Nesse caso, a maioria dos profissionais que apontaram tal aspecto eram pediatras com experiência no atendimento a essas pessoas.

Foi referida ainda por $13 \%$ dos participantes, em sua maioria, pediatras, a importância da identificação precoce para o desenvolvimento de pessoas com autismo, tendo todos esses profissionais experiência no atendimento a autistas. Diante do maior número de pediatras que relataram esse aspecto, enfatiza-se a importância desses profissionais na identificação do transtorno, visto que eles são os primeiros profissionais a entrar em contato com o indivíduo autista.

A regressão, isto é, a perda de habilidades já adquiridas, foi um aspecto referido, quanto ao autismo, por uma fonoaudióloga, uma pedagoga, uma professora, uma neurologista e uma educadora física, todas com experiência com autistas.

\section{Intervenção no autismo e na síndrome de Down}

Com relação à intervenção, $51 \%$ dos participantes apontaram a necessidade de uma equipe de profissionais envolvida no tratamento, o que foi relatado majoritariamente por aqueles com experiência. No que se refere ao autismo, a importância dessa equipe foi citada principalmente pelos profissionais da área médica, da Terapia Ocupacional, da Fonoaudiologia e da Fisioterapia, enquanto, no que diz respeito à síndrome de Down, tal aspecto foi mais frequente no relato dos pedagogos. A maioria dos participantes mencionou que essa equipe deve ser multidisciplinar. A respeito da multidisciplinaridade, Luz (2009) afirma que esse é o modelo dominante nas grandes organizações de saúde; mas os autores do presente artigo destacam a importância de uma atuação interdisciplinar dos profissionais envolvidos no trabalho com pessoas autistas e com síndrome de Down, uma vez que ela envolve não apenas justaposição de saberes, mas a troca de conhecimento entre os profissionais.

Também foi apontado que a intervenção deve considerar as particularidades do indivíduo, seja ele autista ou pessoa com síndrome de Down, o que, no primeiro caso, foi mencionado por $39 \%$ dos profissionais, em sua maioria, pedagogos, psicólogos, terapeutas ocupacionais e professores que tinham experiência com autismo. Com relação à síndrome de Down, tal aspecto foi referido por $32 \%$ dos participantes, tanto por aqueles com experiência quanto pelos sem experiência, tendo sido mais frequente entre os pedagogos. Todos esses profissionais, de uma forma geral, mencionaram que é preciso partir dos interesses dessas pessoas, reconhecendo que, embora elas tenham um diagnóstico de autismo ou de síndrome de Down, ainda assim possuem a sua individualidade.

A intervenção precoce foi referida com uma frequência maior para a síndrome de Down do que para o autismo - por $41 \%$ e por $32 \%$ dos participantes, respectivamente. Entre esses participantes havia um maior número de profissionais que tinham experiência no atendimento a esses indivíduos e eram da área da Terapia Ocupacional. Não obstante, salientase que a intervenção precoce também para o autismo tem sido um aspecto cada vez mais enfatizado como um importante meio para que sejam amenizados os prejuízos decorrentes do transtorno (Fiore-Correia \& Lampreia, 2012). 
Além disso, a grande maioria dos participantes (80\% para o autismo e $83 \%$ para a síndrome de Down) afirmou que a intervenção deve envolver a família, uma vez que é ela quem passa a maior parte do tempo com o indivíduo e por isso deve ser orientada acerca de como lidar com ele. Outro motivo é que o envolvimento da família no tratamento possibilita a troca de informações e a continuidade do trabalho realizado pelos profissionais. Este aspecto foi mencionado dentro de todas as especialidades, tanto por profissionais com experiência quanto por aqueles sem experiência.

Também foram citadas medidas específicas de intervenção como: intervenções grupais, atividades que estimulem a linguagem oral, atividades que trabalhem a coordenação motora e o equilíbrio, psicomotricidade, a Terapia de Integração Sensorial, o Conceito Bobath, terapia com animais, musicoterapia e hidroterapia. No tocante especificamente ao autismo, foi citada ainda como medida interventiva a Metodologia Comportamental, referida por cinco participantes com experiência em autismo - duas pedagogas, uma psiquiatra, um terapeuta ocupacional e uma educadora física. A intervenção medicamentosa também foi citada apenas para o autismo - por $9,3 \%$ do total de participantes -, e somente por profissionais da área médica que tinham experiência no atendimento a autistas.

\section{Inclusão escolar no autismo e na síndrome de Down}

Quanto à inclusão escolar, $89,3 \%$ dos profissionais disseram ser favoráveis à inserção de pessoas com síndrome de Down em escolas regulares, enquanto $77,3 \%$ dos participantes afirmaram tal posicionamento no caso do autismo. Entre esses participantes estavam profissionais de todas as áreas, tanto com quanto sem experiência no trabalho com tais indivíduos; já $16 \%$ dos profissionais, a maioria dos quais não tinha experiência no trabalho com autistas, afirmaram que não sabem ou não se posicionaram claramente se são favoráveis ou desfavoráveis a essa inserção, o que foi mais frequente entre os pedagogos, os professores e os terapeutas ocupacionais. Enquanto isso, no que se refere à síndrome de Down, tal aspecto foi identificado em um número menor de participantes - apenas 9,3\% dos profissionais -, sendo observado no relato de profissionais com e sem experiência no atendimento a indivíduos com síndrome de Down, sobretudo entre os neurologistas. Por sua vez, $6,7 \%$ dos participantes foram desfavoráveis à inserção de pessoas com autismo nas escolas comuns, em sua maioria aqueles da área médica, todos com experiência no trabalho com tais indivíduos. Apenas um educador físico, com experiência, afirmou ser desfavorável à inclusão escolar do indivíduo com síndrome de Down. Dentre as justificativas apresentadas por esses profissionais para a não inserção de indivíduos autistas ou com síndrome de Down nas escolas da rede regular foi mencionada, sobretudo, a possibilidade de que eles sejam vítimas de bullying. Diante desses dados, considera-se importante mencionar o papel da escola na promoção de práticas pedagógicas voltadas ao acolhimento da diversidade humana, com vistas a contribuir para a construção de uma sociedade pautada pelo respeito e aceitação das diferenças.

A necessidade de um preparo maior das escolas foi mencionada, no caso do autismo, por $56 \%$ dos profissionais, e no da síndrome de Down, por $63 \%$ deles, percentual que compreendia tanto aqueles que tinham experiência no trabalho com esses indivíduos quanto os sem experiência. Esse foi um aspecto citado por profissionais de todas as áreas, sendo mais frequente entre os psicólogos.

Parte dos profissionais apontou a necessidade de um auxiliar de sala, havendo uma frequência maior de participantes relatando essa necessidade no caso do autismo, em que foi referida por $13 \%$ dos participantes, em sua maioria pedagogos e tinham experiência no trabalho com autistas. Os profissionais justificaram a necessidade dessa pessoa em virtude dos comportamentos que a criança autista pode apresentar em algumas situações, como as crises de birra, por exemplo. Quanto à síndrome de Down, esse foi um aspecto referido apenas por duas pedagogas, uma fonoaudióloga e um terapeuta ocupacional, todos com experiência no trabalho com pessoas com síndrome de Down; contudo, o último desses profissionais e uma das pedagogas mencionaram que não seria preciso esse auxiliar em todos os casos.

Foi apontada ainda a necessidade de uma parceria entre a escola, a família e os profissionais, mencionada tanto por profissionais que tinham experiência no trabalho com indivíduos autistas e com síndrome de Down quanto por aqueles que nunca haviam 
trabalhado com eles. Tal aspecto foi ressaltado, sobretudo, pelos professores.

Com uma preparação adequada para a inclusão escolar, parte dos profissionais considerou que a inclusão favoreceria o indivíduo com autismo/síndrome de Down e/ou os demais alunos. No tocante à síndrome de Down, os benefícios para aqueles indivíduos, sobretudo o ganho na sociabilidade, foram apontados tanto por profissionais com experiência quanto por inexperientes no trabalho com eles. Quanto ao autismo, tal aspecto foi mencionado por $33 \%$ dos profissionais, que eram principalmente psicólogos, e no tocante à síndrome de Down. Foi citado por $23 \%$ dos profissionais, em sua maioria, fonoaudiólogos e fisioterapeutas. Quanto aos benefícios para os demais alunos que conviveriam com indivíduos autistas ou com síndrome de Down, foi mencionado principalmente o aprendizado com as diferenças.

Ainda quanto à inclusão, $13 \%$ dos profissionais no caso do autismo e $11 \%$ dos participantes no caso da síndrome de Down afirmaram que é preciso considerar o grau de comprometimento do indivíduo. A maioria desses profissionais tinha experiência no atendimento a tais pessoas e era da área da Fisioterapia.

No que se refere especificamente ao autismo, é importante mencionar que três profissionais - uma pediatra, um educador físico e um fisioterapeuta, todos com experiência no atendimento a autistas - apresentaram a concepção de inclusão como sendo integração. Eles entendem que é a criança com autismo quem teria de ser "trabalhada" antes de ser inserida na escola, ou ela é que teria de se adaptar à escola, o que se contrapõe à ideia de inclusão (Lourenço, 2010).

Quanto à inclusão, especificamente, dos indivíduos com síndrome de Down, ainda foi relatado por $13 \%$ dos profissionais que seria mais fácil do que a do indivíduo com autismo. A maior parte deles tinha experiência e era das áreas da Psicologia e da Educação Física.

Alguns profissionais com experiência no trabalho com essas pessoas consideraram a necessidade de colocação do indivíduo com síndrome de Down em uma turma compatível com o seu desenvolvimento; porém houve divergência entre eles quanto a este aspecto, visto que um educador físico e um neurologista mencionaram que essas pessoas deveriam estar junto com indivíduos que tivessem um desenvolvimento cognitivo semelhante. Em contrapartida, duas terapeutas ocupacionais e uma pedagoga consideraram que as pessoas com síndrome de Down precisariam estar em uma turma com indivíduos da sua faixa etária.

Outro aspecto divergente entre determinados profissionais com experiência no atendimento a indivíduos com a síndrome de Down foi a prioridade do ensino formal versus inserção social. Neste sentido, enquanto uma professora e uma terapeuta ocupacional colocaram como prioridade, no contexto da inclusão, a aprendizagem das pessoas com síndrome de Down, outra terapeuta ocupacional, uma pedagoga e um psiquiatra referiram-se à importância da escola primeiramente como um meio de socialização, sendo a aprendizagem, para eles, um fator secundário.

\section{Desafios no autismo e na síndrome de Down}

No que diz respeito aos desafios no trabalho com pessoas com as síndromes aqui estudadas, $24 \%$ dos participantes apontaram, no caso do autismo, a qualificação dos profissionais, a maioria dos quais tinha experiência no trabalho com indivíduos autistas, sendo tal relato mais frequente entre os psicólogos, os profissionais da área médica e os terapeutas ocupacionais, que se referiram principalmente à falta de conhecimento dos profissionais sobre esse transtorno. No tocante à síndrome de Down, esse aspecto foi mencionado por $9 \%$ dos participantes, aí incluídos tanto aqueles com experiência quanto os sem experiência com a síndrome, sendo citado mais frequentemente pelos professores. Considera-se que essa maior frequência de profissionais que apontaram a qualificação dos profissionais em relação ao autismo do que a de profissionais que apontaram a síndrome de Down tem relação com 0 fato de alguns participantes terem considerado que, para o autismo, deveria haver um tratamento específico, com profissionais especializados na área. Ressalta-se ainda que parte dos profissionais da área médica, com experiência no trabalho com pessoas com autismo e com síndrome de Down, falaram sobre a dificuldade de acesso a serviços especializados em órgãos públicos.

Também foi mencionado, por $39 \%$ dos participantes no caso do autismo e por $31 \%$ no caso da síndrome de Down, como um desafio ter que lidar com o indivíduo autista/com síndrome 
de Down. No que concerne ao autismo, esse foi um aspecto relatado por profissionais tanto com quanto sem experiência, sendo prevalente entre os educadores físicos e fisioterapeutas, ao passo que, quando se trata da síndrome de Down, tal aspecto foi mencionado principalmente pelos profissionais com experiência, sobretudo pelos pedagogos; contudo, enquanto no autismo foi abordada a dificuldade interativa, no caso da síndrome de Down, os profissionais referiram-se principalmente às dificuldades em lidar com esses indivíduos devido às limitações cognitivas e comunicativas que podem apresentar.

Outro desafio - citado por $51 \%$ dos participantes no que se refere ao autismo e por $61 \%$ dos profissionais no que diz respeito à síndrome de Down - foi a aceitação, referida por profissionais de todas as especialidades, tanto com quanto sem experiência no trabalho com essas pessoas, sendo prevalente nos relatos dos profissionais da área médica. Esses profissionais referiram-se à aceitação tanto por parte da família quanto da escola e da sociedade em geral.

Também foram apontados desafios relacionados especificamente ao trabalho com a família. A dificuldade relatada com maior frequência foi a falta de continuidade em casa do trabalho realizado pelos profissionais, citada por $36 \%$ dos participantes, no caso do autismo, e por $29 \%$ dos profissionais, no caso da síndrome de Down. Esta foi citada com maior frequência pelos profissionais de Fonoaudiologia que tinham experiência. Foi apontado também como um desafio que envolve o trabalho com a família a superproteção que os pais tendem a oferecer aos filhos autistas ou com síndrome de Down, devido às limitações. A superproteção pode significar também falta de limites.

No que se refere especificamente ao autismo, os profissionais mencionaram também como desafio realizar o diagnóstico $-13 \%$ deles, referido principalmente por psicólogos e pediatras, em sua maioria com experiência no trabalho com indivíduos com autismo. Geralmente esses profissionais mencionaram dificuldade no diagnóstico devido à própria heterogeneidade do transtorno, o que pode ocasionar discordâncias entre os profissionais.

Como a evolução do indivíduo autista com a intervenção muitas vezes é sutil, exigindo um trabalho de longo prazo, $12 \%$ dos participantes em sua maioria psicólogos e psiquiatras com experiência no atendimento a pessoas com autismo - mencionaram como um desafio não desacreditar da eficácia do seu trabalho. Esses profissionais mencionaram ainda que, diante das pequenas evoluções que o indivíduo autista apresenta, inclusive com a possibilidade de haver regressão, esse trabalho é algo que por vezes lhes causa frustração.

Por outro lado, no tocante especificamente à síndrome de Down, alguns profissionais relataram como um desafio ter cuidado com atividades físicas, em virtude dos comprometimentos da síndrome de Down, como as cardiopatias, por exemplo. A necessidade desses cuidados foi referida por dois educadores físicos, uma terapeuta ocupacional e uma fisioterapeuta, todos com experiência no trabalho com indivíduos com síndrome de Down.

\section{CONSIDERAÇÕES FINAIS}

Diante dos resultados deste estudo e daquilo que é abordado pela literatura, percebe-se que, mais de 70 anos após o autismo ter sido descrito pela primeira vez, por Leo Kanner - em um momento histórico de conquista de direitos por parte das pessoas que fazem parte do espectro autista e de seus familiares - pela aprovação da lei que institui a Política Nacional de Proteção dos Direitos da Pessoa com Transtorno do Espectro Autista (Lei $n^{\circ} 12.764$, de 27 de dezembro de 2012), o autismo ainda é pouco conhecido pela população em geral e até mesmo por alguns profissionais entrevistados, como demonstrou o presente estudo. Destacou-se o fato de que os profissionais que desconheciam o autismo eram os que atuavam no contexto escolar, o que traz implicações para a inclusão de crianças e jovens autistas nesses ambientes. Além disso, esse desconhecimento do autismo também pode ser observado nos profissionais que não aceitaram participar do estudo ao saberem que seriam abordadas questões sobre esse transtorno.

Em contrapartida, considera-se que, em relação à síndrome de Down, até por ser uma descoberta mais antiga no campo científico, remontando ao século XIX e tendo já a sua etiologia definida, há um esclarecimento maior a respeito do que se trata, inclusive por meio da mídia, que tem contribuído cada vez mais para a divulgação da síndrome. Considera-se, assim, que muito já se avançou com relação à síndrome de Down, principalmente por meio dos 
progressos na área científica, que têm permitido maior qualidade de vida para essas pessoas.

Aponta-se como uma limitação deste estudo, além da menor quantidade de profissionais da área médica, o fato de não ter sido possível obter um número equivalente de profissionais com e sem experiência no trabalho com pessoas com autismo e com síndrome de Down, visto que houve uma quantidade maior de profissionais experientes. Isso se deu pelo fato de que, em um primeiro momento, muitos profissionais afirmaram não ter experiência em atender essas pessoas, mas no decorrer da entrevista, relataram ter trabalhado em um período anterior ao estudo com esses indivíduos, e assim passaram a fazer parte do grupo de profissionais experientes, já que na presente pesquisa foram consideradas não apenas as experiências atuais dos profissionais, mas também aquelas adquiridas em períodos anteriores. Sugere-se, então, que estudos futuros busquem investigar as concepções de um número maior de profissionais que ainda não trabalharam com indivíduos com autismo ou com síndrome de Down, tendo em vista que essas concepções poderiam subsidiar as suas práticas com tais indivíduos, na hipótese de um dia virem a trabalhar com eles.

Os resultados deste estudo possibilitam refletir sobre a formação dos profissionais, visto que os participantes que afirmaram desconhecer o autismo relataram não ter estudado durante a sua formação disciplinas que abordassem essa temática. Ainda é possível refletir sobre a necessidade de uma maior capacitação dos profissionais para lidar com o autismo e a síndrome de Down, sobretudo quanto à primeira dessas condições, e de investir numa formação profissional que possibilite a construção de práticas embasadas nos documentos legais, tais como a Convenção sobre os Direitos das Pessoas com Deficiência (Decreto $n^{\circ}$ 6.949, de 25 de agosto de 2009), vindo a contribuir para a inclusão escolar e social desses indivíduos.

Por fim, destaca-se, sobretudo, a relevância de estudar concepções, visto que as ações que os indivíduos executam, sejam elas na vida profissional ou pessoal, são influenciadas pelas concepções que eles têm sobre determinado assunto, as quais resultam de um processo histórico. No contexto deste estudo, foi possível conhecer o que fundamenta as práticas de diferentes profissionais em relação ao autismo e à síndrome de Down.

\section{REFERÊNCIAS}

Bardin, L. (1977). Análise de conteúdo. Lisboa: Edições 70.

Baron-Cohen, S. (2008). Autism and Asperger syndrom. Oxford: Oxford University Press.

Camargo, S. P. H., \& Bosa, C. A. (2009). Competência social, inclusão escolar e autismo: revisão crítica da literatura. Psicologia \& Sociedade, 21(1), 65-74.

Castro, G. S., Panhoca, I., \& Zanolli, M. L. (2011). Interação comunicativa em contexto lúdico de duas crianças com síndrome de Down, comportamentos autísticos e privação de estímulos. Psicologia: Reflexão e Crítica, 24(4), 730-738.

Decreto $n^{\circ}$ 6.949, de 25 de agosto de 2009. (2009, 25 de agosto). Promulga a Convenção Internacional sobre os Direitos das Pessoas com Deficiência e seu Protocolo Facultativo, assinados em Nova York, em 30 de março de 2007. Brasília, DF: Presidência da República: Casa Civil. Subchefia para Assuntos Jurídicos. Recuperado em 21 de abril de 2014, de http://www.planalto.gov.br/ccivil_03/_ato20072010/2009/decreto/d6949.htm.

Dumas, J. E. (2011). Psicopatologia da infância e da adolescência. $3^{\mathrm{a}}$ ed. Porto Alegre: Artmed.

Fiore-Correia, O., \& Lampreia, C. (2012). A conexão afetiva nas intervenções desenvolvimentistas para crianças autistas. Psicologia: Ciência e Profissão, 32(4), 926-941.

Goldberg, K. (2002). A percepção do professor acerca do seu trabalho com crianças portadoras de autismo e síndrome de Down: um estudo comparativo. (Dissertação de Mestrado). Instituto de Psicologia, Universidade Federal do Rio Grande do Sul, Porto Alegre.

Griffith, G. M., Hastings, R. P., Nash, S., \& Hill, C. (2010). Using matched groups to explore child behavior problems and maternal well-being in children with Down syndrome and autism. Journal of Autism and Developmental Disorders, 40(5), 610-619.

Heidgerken, A. D., Geffken, G., Modi, A., \& Frakey, L. (2005). A survey of autism knowledge in a health care setting. Journal of Autism and Developmental Disorders, 35(3), 323-330.

Henn, C. G., Piccinini, C. A., \& Garcias, C. L. (2008). A família no contexto da síndrome de Down: revisando a literatura. Psicologia em Estudo, 13(3), 485-493.

Imran, N., Chaudry, M. R., Azeem, M. W., Bhatti, M. R., Choudhary, Z. I., \& Cheema, M. A. (2011). A survey of autism knowledge and attitudes among the healthcare professionals in Lahore, Pakistan. BMC Pediatrics, 11(107), 1-21.

King, G., Baxter, D., Rosenbaum, P., Zwaigenbaum, L., \& Bates, A. (2009). Belief systems of families of children with autism spectrum disorders or Down 
syndrome. Focus on Autism and Other Developmental Disabilities, 24(1),50-64.

King, G. A., Zwaigenbaum, L., King, S., Baxter, D., Rosenbaum, P., \& Bates, A. (2006). A qualitative investigation of changes in the belief systems of families of children with autism or Down syndrome. Child: Care, Health and Development, 32(3), 353369.

Klin, A. (2006). Autismo e síndrome de Asperger: uma visão geral. Revista Brasileira de Psiquiatria, 28(Suppl.1), 3-11.

Klin, A., \& Mercadante, M. T. (2006). Autismo e transtornos invasivos do desenvolvimento. Revista Brasileira de Psiquiatria, 28(Suppl. 1), 1-2.

Lampreia, C., \& Lima, M. M. R. (2008). Instrumento de vigilância precoce do autismo: manual e vídeo. Rio de Janeiro: PUC-Rio; São Paulo: Loyola.

Lei $n^{\circ} 12.764$, de 27 de dezembro de 2012. (2012, 27 de dezembro). Institui a Política Nacional de Proteção dos Direitos da Pessoa com Transtorno do Espectro Autista; e altera o § 3ㅇ do art. 98 da Lei $n^{\circ} 8.112$, de 11 de dezembro de 1990. Brasília, DF: Presidência da República: Casa Civil. Subchefia para Assuntos Jurídicos. Recuperado em 20 de janeiro de 2013, de http://www.planalto.gov.br/ccivil_03/_ato20112014/2012/lei/l12764.htm.

Lourenço, E. (2010). Conceitos e práticas para refletir sobre a educação inclusiva. Série Cadernos da Diversidade. Belo Horizonte: Autêntica; Ouro Preto: UFOP.

Luz, M. T. (2009). Complexidade do campo da saúde coletiva: multidisciplinaridade, interdisciplinaridade e transdisciplinaridade de saberes e práticas análise sócio-histórica de uma trajetória paradigmática. Saúde e Sociedade, 18(2), 304311.

Melo-de-Aguiar, A. (2009). O desenvolvimento e a criança com síndrome de Down. In M. L. Seidl-deMoura, D. M. L. F. Mendes, \& L. F. Pessôa, (Eds.). Interação social e desenvolvimento (pp. 177-189). Curitiba: CRV.
Mitchell, P., \& O'Keefe, K. (2008). Brief report: do individuals with autism spectrum disorder think they know their own minds? Journal of Autism and Developmental Disorders, 38(8), 1591-1597.

Moreira, L. M. A., \& Gusmão, F. A. F. (2002). Aspectos genéticos e sociais da sexualidade em pessoas com síndrome de Down. Revista Brasileira de Psiquiatria, 24(2), 94-99.

Nunes, M. D. R., \& Dupas, G. (2011). Independence of children with Down syndrome: the experiences of families. Revista Latino-Americana de Enfermagem, 19(4), 985-993.

Pisula, E. (2007). A comparative study of stress profiles in mothers of children with autism and those of children with Down's syndrome. Journal of Applied Research in Intellectual Disabilities, 20(3), 274-278.

Reilly, C. (2009). Autism spectrum disorders in Down syndrome: A review. Research in Autism Spectrum Disorders, 3(4), 829-839.

Rodrigues, E. C., Alchieri, J. C., \& Coutinho, M. P. L. (2010). A afetividade de crianças e jovens com síndrome de Down: um estudo sobre as percepções de pais e de professores. Revista CES Psicología, 3(2), 79-98.

York, A., Fraunhofer, N. von, Turk, J., \& Sedgwick, P. (1999). Fragile-X syndrome, Down's syndrome and autism: awareness and knowledge amongst special educators. Journal of Intellectual Disability Research, 43(3), 314-324.
Recebido em 22/07/2013 Aceito em 12/05/2014

Cibele Shírley Agripino-Ramos: psicóloga, mestre em Psicologia Social pela Universidade Federal da Paraíba.

Nádia Maria Ribeiro Salomão: doutora em Psicologia pela University of Manchester - Inglaterra, e pós-doutorado pela University of North Carolina - EUA; professora do Departamento de Psicologia e do Programa de Pós-Graduação em Psicologia Social da Universidade Federal da Paraíba. 